\title{
Communs (im)matériels : enjeux épistémologiques, institutionnels et politiques
}

Introduction au dossier « Communs (im)matériels. Conjuguer les dimensions matérielles et immatérielles des communs »

(In)tangible commons : epistemological, institutional and political issues

Special section: "(In)tangible commons. Combining the tangible and intangible

dimensions of the commons"

Arnaud Buchs, Catherine Baron, Géraldine Froger et Adrien Peneranda

\section{(2) OpenEdition}

\section{Édition électronique}

URL : http://journals.openedition.org/developpementdurable/13701

DOI : 10.4000/developpementdurable.13701

ISSN : 1772-9971

Éditeur

Association DD\&T

Référence électronique

Arnaud Buchs, Catherine Baron, Géraldine Froger et Adrien Peneranda, « Communs (im)matériels : enjeux épistémologiques, institutionnels et politiques », Développement durable et territoires [En ligne], Vol. 10, n¹ | Avril 2019, mis en ligne le 04 avril 2019, consulté le 01 mai 2019. URL : http:// journals.openedition.org/developpementdurable/13701; DOI : 10.4000/developpementdurable.13701

Ce document a été généré automatiquement le 1 mai 2019.

Développement Durable et Territoires est mis à disposition selon les termes de la licence Creative Commons Attribution - Pas d'Utilisation Commerciale 4.0 International. 


\section{Communs (im)matériels : enjeux épistémologiques, institutionnels et politiques}

Introduction au dossier « Communs (im)matériels. Conjuguer les

dimensions matérielles et immatérielles des communs »

(In)tangible commons : epistemological, institutional and political issues

Special section: "(In)tangible commons. Combining the tangible and intangible

dimensions of the commons"

Arnaud Buchs, Catherine Baron, Géraldine Froger et Adrien Peneranda

Ce dossier thématique est pour partie le fruit de travaux réalisés dans le cadre du LABEX SMS, portant la référence ANR-11-LABX-0066. Il a bénéficié d'une aide de l'État gérée par l'Agence nationale de la recherche au titre du programme Investissements d'avenir portant la référence ANR-11-IDEX-0002-02.

Nous tenons à remercier les collègues ayant accepté d'évaluer les contributions qui composent ce dossier.

1 L'ambiguïté et la polysémie de la notion de « communs » (comme systèmes de ressources, comme systèmes de valeurs ou comme principe d'action politique) expliquent qu'elle fasse l'objet d'approches aux objectifs scientifiques et/ou politiques hétérogènes (Nahrath, 2015). L'engouement suscité par cette notion dans les arènes académiques et citoyennes se traduit par un nombre croissant de publications depuis le début des années 2000, surtout depuis l'attribution du «Nobel d'économie » à Elinor Ostrom en 2009 (figure 1) ${ }^{1}$. Ne serait-ce qu'en 2018 pour ce qui concerne la littérature en français, nous pouvons citer la parution du numéro spécial de la revue Responsabilité \& environnement intitulé «Les communs environnementaux: gérer autrement la rareté », celle du numéro 233 de la Revue internationale des études du développement à propos de la gouvernance des communs, ou encore celle du numéro de la revue Espaces et Sociétés consacré aux enjeux territoriaux des communs. 
Figure 1. Les communs : un nombre croissant de publications

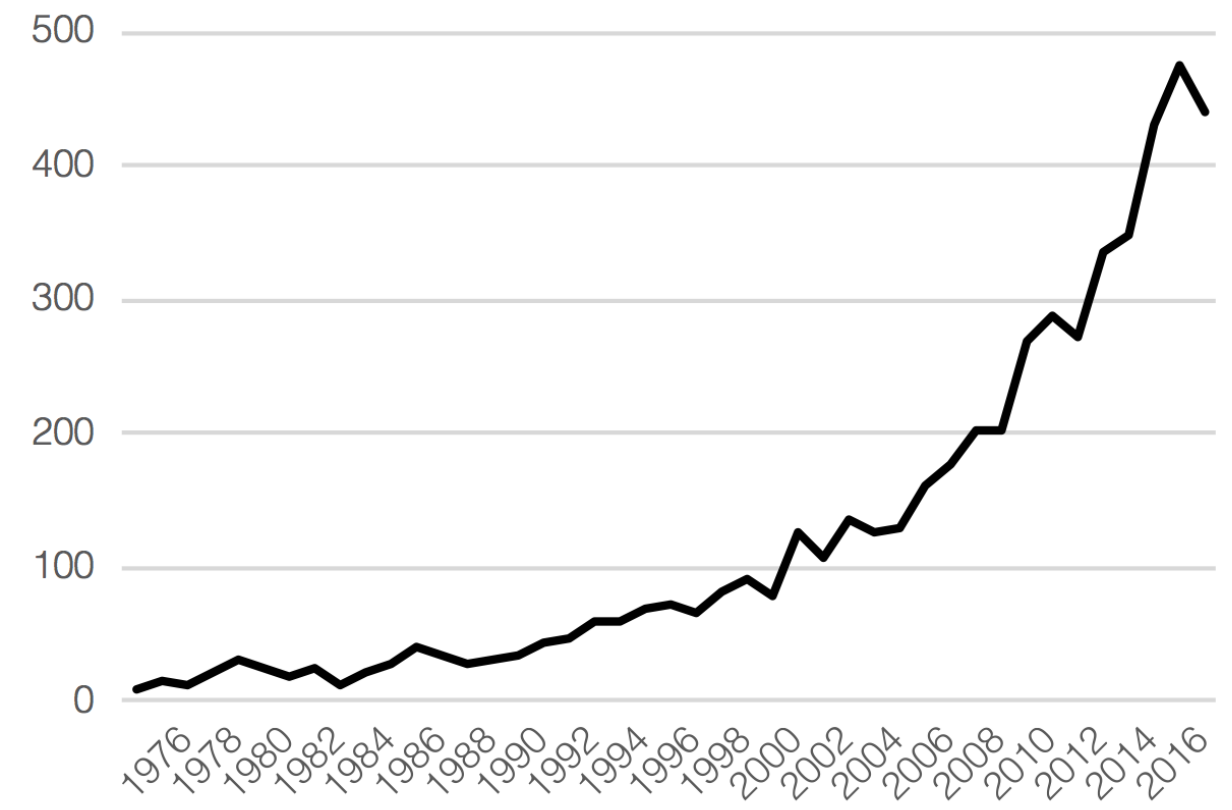

Note : Recherche à partir de Web of Science Core Collection. Requête réalisée le 1/12/2018 : TITLE : ("commons") OR TOPIC : ("the commons") OR TOPIC : ("common pool resource") OR TOPIC :

("common pool resources") OR TOPIC : ("common resource") OR TOPIC : ("common resources") OR TOPIC : ("common property management") OR TOPIC : ("common property institution") OR TOPIC : ("common property institutions") OR TOPIC : ("anticommons") NOT TOPIC : ("house of commons") ; Timespan : 1975-2017. Résultats totaux : 5273

Source : les auteurs

2 Une publication supplémentaire sur les communs pourrait sembler superflue. Pourtant, ce dossier se démarque des précédents par un constat, mis en lumière lors de la journée d'étude à son origine ${ }^{2}$ : la littérature académique sur les communs est le plus souvent approchée par des objets spécifiques et partitionnée entre, d'une part, les approches des communs comme objets matériels (communs environnementaux, systèmes d'irrigation, forêts, ressources halieutiques, coopératives, etc.) et, d'autre part, les communs immatériels, également dénommés communs informationnels ou communs de la connaissance [knowledge commons] (brevets, savoirs et connaissances, indications géographiques, open source, culture, etc.) (Hess et Ostrom, 2003 ; 2007 ; Frischmann et al., 2014). Rares sont les travaux qui discutent le lien entre les dimensions matérielles et immatérielles des communs, ce que propose de faire ce dossier thématique de la revue Développement durable et territoires. Autrement dit, il s'agit d'affiner la compréhension de la dimension immatérielle des communs matériels et/ou de la dimension matérielle des communs immatériels, voire de montrer que cette dichotomie est illusoire. Ce dossier fait ainsi écho aux travaux antérieurs visant à comprendre l'articulation entre le matériel et l'idéel (Godelier, 2010) en montrant comment les réalités matérielles agissent sur l'organisation et les dynamiques sociales, et inversement. L'étude des communs ne peut se réduire à la nature de "biens", fonction de caractéristiques intrinsèques, ni à un "régime de propriété » régulant leurs usages et favorisant un mode de gouvernance spécifique, ni même à des situations particulières dont la compréhension se limiterait à des analyses locales. La portée générale des communs participe de la complexité de la vie sociale en prise avec la grande variété des formes d'action collective. Ces dernières 
relèvent, la plupart du temps, de dimensions privées, publiques et communes qui s'imbriquent, si bien qu'il est difficile de délimiter avec précision les contours des communs (Harribey, 2011; Cole et Ostrom, 2012). Néanmoins, elles incitent à questionner la possibilité d'une alternative au-delà du marché et de l'État (Ostrom, 2010).

3 Afin de traiter cette entrée originale, l'appel à contributions encourageait les approches pluri, voire inter-disciplinaires, ainsi que le croisement des dimensions théoriques et empiriques (via des études de cas tant dans les pays du Nord que du Sud). Il avait pour ambition de questionner: (i) les défis théoriques et analytiques posés par l'articulation des dimensions matérielles et immatérielles des communs ; (ii) les dynamiques sociales en matière de régulation et de territorialisation de la gouvernance des communs; (iii) les enjeux de soutenabilité (environnementale, sociale, économique, territoriale, etc.). Nous postulions que, loin d'être cantonnés à des pratiques séculaires (notamment pour la régulation des ressources), les communs sont au contraire susceptibles de participer d'innovations sociales.

4 Les articles publiés dans ce dossier répondent tout à fait à ces attentes ${ }^{3}$. À l'étendue des objets abordés (l'espace urbain, l'eau, le territoire, les coopératives, la culture, les semences ou encore les estives) s'ajoute une variété de corpus théoriques et conceptuels, reflet d'une grande diversité disciplinaire (économie, géographie, aménagement, droit, gestion, anthropologie et sciences politiques).

Ces contributions révèlent que la conjugaison des dimensions matérielles et immatérielles est indispensable pour rendre compte de la complexité inhérente aux communs. Dans cette introduction, nous montrons que cette conjugaison peut être appréhendée via trois entrées, non exclusives les unes des autres, puisqu'elles procèdent toutes de cette complexité. La première entrée, épistémologique, questionne la possibilité de circonscrire les communs. L'a priori selon lequel un objet particulier considéré comme un commun pourrait, par essence, être matériel ou immatériel, est remis en question à partir d'une clarification des définitions et des niveaux d'analyse des communs. La deuxième entrée, institutionnelle, articule les dimensions matérielles et immatérielles des communs par les enjeux liés à la définition/adoption de règles, en particulier celles relatives à la propriété, à la possession et à l'accès, mais également par les jeux d'acteurs qui y sont associés. La troisième entrée appréhende les liens entre les dimensions matérielles et immatérielles par les enjeux politiques inhérents aux communs, en insistant moins sur les communs comme systèmes de ressources, que comme supports d'un agir politique.

\section{Les communs comme objets complexes à délimiter : enjeux épistémologiques}

6 Ce dossier thématique n'a pas pour objet de revenir sur l'émergence de la problématique des communs, déjà bien détaillée dans la littérature ${ }^{4}$ et rappelée de manière synthétique ci-dessous. Notre premier objectif est d'insister sur leur complexité intrinsèque qui participe pour beaucoup à leur intérêt.

7 Formellement apparue dans les années 1980 avec la création de l'International Association for the Study of Common Property en $1989^{5}$, l'École de Bloomington (dont la figure emblématique est Elinor Ostrom), axée sur la gouvernance des systèmes de ressources communes [common-pool resources], conteste la thèse de Garrett Hardin (1968) qui 
n'identifiait que deux solutions pour se prémunir de la tragédie qui menacerait les communs : l'appropriation privée ou le contrôle direct par la puissance publique. La collection de nombreuses études de cas (sur différentes ressources, dans de multiples pays) et leur mise en débat par de nombreux auteurs (se réclamant ou non de l'École de Bloomington) ont permis, d'une part, d'affirmer la diversité institutionnelle des modes de gouvernance des ressources basés sur l'auto-organisation et, d'autre part, d'identifier les critères d'analyse et de succès des modes de gestion de systèmes de ressources communes (Wade, 1988 ; Ostrom, 1990 ; Baland et Platteau, 1996).

Ces travaux mettent en lumière la capacité des systèmes relevant de communs à modifier la gestion des ressources afin d'assurer leur préservation via des mécanismes d'incitations et de contraintes. Ils ont participé au développement de problématiques plus générales en sciences sociales relatives à la compréhension des dynamiques d'action collective, par la prise en compte des normes informelles et de la dimension politique du changement institutionnel (Agrawal, 2003).

Malgré la profusion des recherches, des confusions obscurcissent les débats (Ostrom, 2000 ; Weinstein, 2013; Sarker et Blomquist, 2018). Elles concernent notamment la différence entre : (1) régimes de propriété commune et libre accès (la confusion originelle de Garrett Hardin), (2) systèmes de ressources communes [common-pool resources (CPR)] et régimes de propriété commune [common-property regimes] (Ciriacy-Wantrup et Bishop, 1975 ; Bromley, 1989) et (3) systèmes de ressources et flux d'unités de ressources [flow of resource units] $]^{6}$. Ce dernier point a été exploré par Charlotte Hess et Elinor Ostrom (2003 : 112) dans leurs travaux sur l'information comme système de ressources communes. Les auteures relèvent que l'information elle-même pouvait être «de plus en plus privatisée, surveillée, cryptée et restreinte ${ }^{7} »$. Elles insistent alors sur la nécessité de distinguer l'infrastructure (une bibliothèque, un registre, Internet), l'artefact (un livre, un fichier informatique, une page web) et l'idée (la connaissance, l'information, une donnée). Si l'on opère ces distinctions analytiques, «le concept de commun ne renvoie strictement ni à un ensemble de ressources, ni à ce qui dérive de cet ensemble (valorisation du flux), ni à une communauté, ni à un régime de propriété, ni à des infrastructures ou à un lieu, mais à l'arrangement institutionnel de ces éléments contingent à un contexte (d'autres arrangements étant observables)»(Allaire, 2013). Il n'y a pas de correspondance stricte entre les catégories de biens et les régimes de propriété : «Les systèmes de ressources dites communes peuvent être détenus par des gouvernements locaux, régionaux ou nationaux; par des communautés; par des individus privés ou des entreprises; ou être utilisés librement par quiconque pouvant y avoir accès » (Ostrom, $2000: 338)^{8}$.

Dès lors, comment définir et délimiter un commun ${ }^{9}$ ? La manière la plus courante d'appréhender les communs consiste à se référer à la typologie permettant de distinguer quatre types de biens en fonction de deux critères, l'exclusion et la « soustractibilité $»^{10}$ : l'exclusion renvoie à la possibilité d'exclure des bénéficiaires potentiels d'un bien ou d'un service. La soustractibilité (ou rivalité dans la consommation/usage) qualifie la possibilité qu'un usage ou que la consommation d'une personne restreigne en quantité ou en qualité la disponibilité d'un bien ou d'un service pour l'usage ou la consommation d'autres personnes (possibilité ou non d'usage ou de consommations simultanés) (Ostrom, 2005 : 23) ${ }^{11}$. 
Tableau 1. Quatre types de biens

\begin{tabular}{|c|c|c|c|}
\hline & & \multicolumn{2}{|c|}{ Soustraction de la ressource à l'usage d'autrui } \\
\hline & & Élevée & Faible \\
\hline \multirow{2}{*}{$\begin{array}{l}\text { Difficulté } \\
\text { d'exclusion des } \\
\text { bénéficiaires } \\
\text { potentiels }\end{array}$} & Élevée & $\begin{array}{l}\text { Ressources communes: } \\
\text { bassins d'eau souterraine, } \\
\text { lacs, systèmes d'irrigation, } \\
\text { pêcheries, forêts, etc. }\end{array}$ & $\begin{array}{l}\text { Biens publics : paix et sécurité de la } \\
\text { communauté, défense nationale, } \\
\text { connaissances, protection contre les } \\
\text { incendies, } \\
\text { météorologiques, etc. }\end{array}$ \\
\hline & Faible & $\begin{array}{l}\text { Biens privés: } \\
\text { alimentation, vêtements, } \\
\text { automobiles, etc. }\end{array}$ & $\begin{array}{l}\text { Biens de péage (de club): théâtres, } \\
\text { clubs privés, garderies, etc. }\end{array}$ \\
\hline
\end{tabular}

Source : Ostrom (2010 : 645)

11 Cette typologie des biens a fait l'objet de nombreuses critiques. Dans une perspective socioconstructiviste, Stéphane Nahrath (2015) en formule deux. D'une part, cette typologie témoigne d'une conception statique et substantialiste des biens. L'auteur énonce que la nature des biens résulte moins de leurs caractéristiques "intrinsèques » que de l'évolution de la demande (critère de rivalité ou de soustractibilité) et des régimes de propriété qui en régulent l'accès et l'usage (critère d'exclusion) : « Les théoriciens des CPR peinent à admettre que la nature d'un bien ou d'une ressource - et notamment les biens communs - résulte en premier lieu d'un processus de construction sociale et politique qui s'opère à travers la mise en place du régime de propriété. Ainsi, les biens communs sont moins des "faits de nature", que des "faits d'institution", c'est-à-dire des constructions sociales et politiques " (Nahrath, 2015 : 77).

D'autre part, des travaux contemporains soulignent la sous-estimation de la porosité (voire la confusion) entre les biens communs et les biens de club, lesquels sont caractérisés par des mécanismes d'exclusion et de délimitation des ayants droit. Par exemple, dans le cas de systèmes d'irrigation communautaires en Suisse (les bisses) objet de l'article séminal de Robert McC. Netting (1974) -, Rémi Schweizer (2018) montre que la limitation du nombre d'ayants droit (les ins) est une des conditions de la création et de la stabilisation du régime institutionnel régulant la ressource et les infrastructures. De manière symétrique, l'exclusion de certains usagers (les outs), dont l'importance est souvent sous-estimée, contribue à l'évolution de biens considérés comme communs en biens de club (voire en biens privés). À ce titre, Jean-Marie Harribey (2011) montre que les communs sont loin d'être consensuels; au contraire, ils sont un espace de rapports de pouvoir. Les analyser de ce point de vue conduit à interroger la représentativité et la légitimité des acteurs tout comme les dynamiques internes à ces formes collectives (Leyronas et Bambridge, 2018). Ceci illustre la porosité de nos trois entrées, épistémologique, institutionnelle et politique.

13 L'entrée épistémologique est sous-jacente à la plupart des articles de ce dossier thématique. Néanmoins, trois d'entre eux l'abordent de façon explicite. La complexité et la difficulté à circonscrire les communs sont des problématiques traitées par Gilles Allaire (2019) en termes d'ambivalence des communs. Après avoir clarifié diverses notions, en 
particulier celles développées par Elinor Ostrom, et mis en lumière les sources de confusion, l'auteur propose des éléments de compréhension pour saisir cette complexité, liée pour une large part à des enjeux de temporalité. Pour ce faire, il mobilise les apports de John R. Commons pour conceptualiser la dimension intangible, immatérielle, des communs. Afin de saisir les communs en dynamique, trois niveaux d'analyse sont distingués. Le premier, qualifié de positif, renvoie aux systèmes de ressources collectives. Le deuxième est celui du bien commun, vu comme un ensemble de valeurs et d'idéaux qui animent l'action collective. Le troisième relève des communs en tant que forme d'organisation politique. L'ambivalence des communs résulterait alors de leur complexité institutionnelle et de la dimension temporelle et instituante du social.

Le caractère dynamique des communs est au cœur de l'article de Nicolas Buclet et Juliette Cerceau (2019); ils ajoutent à la dimension temporelle la dimension territoriale. En s'appuyant sur le cas du système agricole d'Aussois (Savoie), les auteurs mobilisent les apports de l'écologie territoriale, notamment l'approche par le métabolisme, pour conjuguer en dynamique les dimensions matérielles et immatérielles des systèmes de ressources communes. Leur approche originale du métabolisme territorial leur permet de saisir la circulation des flux et de dépasser une scission arbitraire entre matériel et immatériel pour un territoire donné. Reste à expliquer la raison d'être de ce métabolisme, étant entendu que les ressources (matérielles et immatérielles) sont elles-mêmes des construits spatialisés. Pour ce faire, ils couplent la notion « d'agir commun », empruntée à Pierre Dardot et Christian Laval (2014), avec celle de «capabilité territoriale » faisant écho à la notion développée par Amartya Sen (2000). La grille d'analyse proposée, mobilisable sur d'autres cas, leur permet également d'appréhender les enjeux de soutenabilité.

L'article de Fabien Girard (2019) à propos des semences et de l'agrobiodiversité démontre, pour sa part, la nécessité d'une re-conceptualisation du cadre des communs du végétal en étudiant les problèmes issus de leur réduction à des ressources génétiques, même quand celles-ci sont accessibles à des communautés locales. L'auteur propose une nouvelle lecture épistémologique des bio-communs locaux, à même de rendre visibles les réseaux semenciers traditionnels et la complexité des cadres sociaux préexistants. Il préconise de limiter la rationalisation de l'action collective induite par la mise en œuvre de politiques publiques et la réglementation qui vise à améliorer la traçabilité et la certification des semences. L'analyse des relations entre communautés et institutions, puis entre communautés et ressources, permet une meilleure compréhension des dynamiques de circulation des semences. Ce faisant, l'auteur traite également des enjeux institutionnels, au cœur du point suivant.

\section{Les communs comme relations : enjeux institutionnels et jeux d'acteurs}

16 La deuxième entrée, institutionnelle, conjugue les dimensions matérielles et immatérielles des communs par les enjeux liés à la définition et l'adoption de règles, en particulier celles relatives à la propriété, à la possession et à l'accès, mais également par les jeux d'acteurs qui y sont relatifs.

17 L'émergence d'une approche institutionnaliste en économie témoigne d'une rupture dans l'histoire de la pensée: elle se propose de renouveler l'objet sur lequel doit porter la 
science économique par la prise en compte des règles au sens large, dimension immatérielle de la coordination. Elinor Ostrom introduit cette dimension dans l'analyse de la gouvernance des ressources pour comprendre les règles qui les instituent comme communs. Mais son approche reste figée dans une vision «stratégique » des règles (ses travaux étant ancrés dans la «nouvelle économie institutionnelle »). Les apports de John R. Commons (2005 [1934]) permettent de se démarquer d'une telle approche. L'auteur propose de s'émanciper d'une conception matérialiste adossée à la catégorie de marchandise adoptée par la majorité des économistes (classiques et hédonistes). Il lui substitue une théorie de l'activité «fondamentalement relationnelle» (Chavance, 2012:35) en prenant les "trans-actions" pour unité élémentaire de la recherche économique. Celles-ci "ne sont pas un "échange de marchandises" au sens physique de "distribution", mais sont l'aliénation et l'acquisition, entre les individus, des droits de propriété future sur les objets physiques, tels que définis par les règles opérantes collectives de la société. Le transfert de ces droits doit en outre être négocié entre les parties concernées, tel que prévu par les règles opérantes de la société, avant même que le travail ne produise, que les consommateurs ne consomment, ou que les marchandises ne soient physiquement échangées ${ }^{12} »$. (Commons, 2005 [1934] : 58).

Se focaliser sur les institutions, et donc sur la coordination, implique d'affirmer la prise en compte des dimensions immatérielles. C'est le cas par exemple si l'on considère la double fonction des institutions identifiée par Thorstein Veblen (un des précurseurs des approches institutionnalistes avec John R. Commons) et reprise par Marc R. Tool (1977 : 837) : d'une part, les fonctions instrumentales relatives à la dimension téléologique des institutions (habiliter et contraindre des activités); d'autre part, les fonctions cérémoniales relatives à l'appartenance à des groupes, à l'affirmation de statuts et aux relations de pouvoir.

Parce qu'elle traite de la coordination et des règles autour de la gestion de ressources, la littérature sur les communs peut être considérée comme une application particulière d'approches institutionnalistes qui ne se limitent pas à la «nouvelle économie institutionnelle ». Elle appelle à questionner les catégories usuelles du droit relatives à la propriété et à l'usage.

20 C'est le cas de l'analyse en termes de « faisceaux de droits » [bundles of rights] (déjà mis en lumière par John R. Commons en 1934) pouvant être détenus par des utilisateurs de ressources (Schlager et Ostrom, 1992; Ostrom et Schlager, 1996). En montrant que la propriété ne se limite pas à l'aliénation, il devient possible de distinguer et de caractériser les différents régimes de propriété - publique, privée, commune et libre accès - en révélant la diversité institutionnelle potentielle et la variété des détenteurs de droits pouvant coexister au sein d'un régime de propriété (Orsi, 2013). Cinq ensembles de droits sont identifiés. Ils sont relatifs à l'accès (à une ressource, un espace, une infrastructure) ; au prélèvement (extraction); à la gestion (définition des moyens d'extraire et de transformer la ressource); à l'exclusion (capacité à déterminer les ayants droit et modalités de transfert de droits); et à l'aliénation (capacité à vendre les droits de gestion et d'exclusion). Des travaux ultérieurs sur les communs immatériels et informationnels (en particulier liés à Internet) les complètent par deux autres types de droits : celui de contribuer à l'évolution d'un contenu et celui de retirer des éléments obsolètes (Hess et Ostrom, 2007). 
Tableau 2. Faisceaux de droits associés aux positions des acteurs

\begin{tabular}{|l|l|l|l|l|l|}
\hline & Owner & Proprietor & Claimant & Authorized user & $\begin{array}{l}\text { Authorized } \\
\text { entrant }\end{array}$ \\
\hline Access & $\mathrm{X}$ & $\mathrm{X}$ & $\mathrm{X}$ & $\mathrm{X}$ & $\mathrm{X}$ \\
\hline Withdrawal & $\mathrm{X}$ & $\mathrm{X}$ & $\mathrm{X}$ & $\mathrm{X}$ & \\
\hline Management & $\mathrm{X}$ & $\mathrm{X}$ & $\mathrm{X}$ & & \\
\hline Exclusion & $\mathrm{X}$ & $\mathrm{X}$ & & & \\
\hline Alienation & $\mathrm{X}$ & & & & \\
\hline
\end{tabular}

Sources : Ostrom et Schlager (1996: 133) ; Ostrom (2000 : 340) ${ }^{13}$

21 Au-delà d'une approche en termes de règles et de droits, l'action collective renvoie à des jeux d'acteurs qu'il importe de décoder. Pris comme systèmes (ou architectures) de ressources [common-pool resources], les communs produisent des flux de ressources, supports d'activités humaines, qui débordent bien souvent à la fois l'enjeu premier d'un commun particulier, mais également le groupe de bénéficiaires directs. Par exemple, Masahiko Aoki (2006) montre en quoi la fourniture d'eau n'est pas le seul enjeu de l'action collective autour d'un réseau d'irrigation collectif. L'incitation à collaborer au fonctionnement d'un commun (renvoyant ici à la mobilisation et à la répartition de l'eau d'irrigation) est également liée au degré d'adhésion à une croyance partagée relative à la menace d'ostracisme social (exclusion de la communauté villageoise pour les autres décisions). L'appartenance au groupe, sa pérennité et son évolution, les enjeux liés aux savoirs (partagés ou non) sur les ressources et les moyens techniques et sociaux pour les exploiter et les répartir (Aubriot et Riaux, 2013), ou encore le rôle du capital social (en lien avec la confiance mutuelle) (Ballet, 2007), sont autant de dimensions immatérielles liées à un commun, à première vue, matériel. Ces éléments exercent une influence prépondérante sur les modalités d'action collective (par exemple lorsqu'ils visent à réduire les comportements opportunistes) et peuvent constituer à leur tour des ressources mobilisables par les acteurs. D'une manière symétrique, les communs immatériels, ou "communs de l'environnement culturel» selon l'expression de Brett M. Frischmann (2012), sont à considérer comme des supports nécessaires pour des activités matérielles, notamment les activités productives (Allaire, 2013). Comme le montre la littérature sur les labels, les indications géographiques ou les données (big data), les dimensions matérielles et immatérielles des communs questionnent les catégories de collectif, de territoire, de patrimoine ou encore de sécurité (Linck, 2012 ; Calvo-Mendieta et al., 2017).

Deux articles du dossier thématique analysent explicitement (mais pas seulement) ces enjeux. Celui d'Isabelle Bagdassarian, Adrien Peneranda et Catherine Baron (2019) traite d'un objet canonique de la littérature sur les communs : les pâturages d'altitude collectifs (les estives). Les auteurs mobilisent une grille d'analyse interdisciplinaire au croisement de l'aménagement du territoire, des sciences de gestion et de l'économie institutionnelle. Ils révèlent l'enchevêtrement des dimensions matérielles et immatérielles des estives 
dont la gestion repose sur des représentations partagées, une distribution de droits d'usage implicites et des règles d'action collective dans le cadre de collectifs organisés autour du pastoralisme transhumant. Les auteurs notent que la gestion collective de ces biens indivis relève de plusieurs registres de règles et de niveaux de formalisation de celles-ci. Ils questionnent également la pérennité des estives en Pyrénées-Atlantiques qui articulent projets collectifs et intérêts privés des acteurs, dans un contexte marqué par plusieurs communautés gestionnaires des biens et des usages en évolution.

En s'inscrivant dans la continuité de travaux proposant d'appréhender l'entreprise comme un " commun " (Gand et Segrestin, 2009 ; Dacheux et Goujon, 2013; Bommier et Renouard, 2018), Yvan Renou et Hervé Charmettant (2019) analysent les processus de transformation coopérative renvoyant à la création de sociétés coopératives et participatives (SCOP) via la transmission ou la reprise d'entreprises «classiques». Ils mobilisent les apports de l'École de Bloomington ainsi que ceux de Dardot et Laval (2014) dans une approche relevant d'un institutionnalisme sociologique qu'ils appliquent à l'étude de la transformation de six entreprises de la région Auvergne-Rhône-Alpes. Les auteurs appréhendent la transformation coopérative comme un " travail de négociation " entre parties prenantes autour d'un système de ressources portant sur un engagement financier partagé (les «biens communs »), l'élaboration de finalités légitimes pour tous ("un projet commun»), le tout découlant d'une action collective dans un cadre démocratique ( l'agir commun»). Ce faisant, ils illustrent les tensions posées par l'articulation des dimensions matérielles et immatérielles de ce système et évaluent la soutenabilité des sociétés coopératives et participatives. Les auteurs vont au-delà des seuls enjeux institutionnels liés à la "transformation coopérative» (enjeux de gouvernance démocratique). Ils considèrent que les Scop donnent sens à un "projet commun" comprenant des objectifs alternatifs (la revitalisation d'un territoire, la promotion de produits agroalimentaires d'origine locale et/ou issus de l'agriculture biologique, le renforcement du lien social...). Il s'agit le plus souvent de contrer les dérives des entreprises capitalistes multinationales. À partir d'études de cas, les auteurs relatent des expériences réussies et d'autres qui ont échoué à articuler les dimensions économiques (viabilité d'une activité et emplois) et politiques.

\section{Les communs comme projet : enjeux politiques}

La catégorie de commun fait intervenir des réflexions de philosophie politique en termes de justice et d'équité. Elle fait écho aux débats relatifs à la valeur intrinsèque d'objets tels que la biodiversité (Larrère et Larrère, 2015) ou à la reconnaissance de l'eau comme patrimoine commun (Petit et Romagny, 2009). Ces différents exemples, loin d'être exhaustifs, constituent autant de pistes permettant de questionner les liens entre les dimensions matérielles et immatérielles des communs du point de vue politique.

Il subsiste a minima une source de confusion liée à la polysémie des termes. Il nous semble nécessaire de distinguer, d'une part, les communs en tant que systèmes de ressources et/ ou architectures institutionnelles liées à la répartition de ressources et, d'autre part, le « bien commun » en tant que système de valeurs qui oriente l'action collective et qui peut être interprété comme un principe d'action politique (Allaire, 2013). Le bien commun comme principe d'action politique a une généalogie riche dans l'histoire de la pensée occidentale, depuis la recherche de "l'avantage commun" [koinê sumpheron] chez Aristote, notion traduite par « utilité commune » [utilitas communis] par Cicéron, jusqu'au 
bonum commune de Thomas d'Aquin. Dans cette tradition, le bien commun devient le « fondement éthique du politique » qui renvoie à la recherche de l'intérêt commun de la cité comme principe et finalité du politique (Dardot et Laval, 2014:26-28). Si le caractère politique du commun est évident pour le second niveau d'analyse, il est également prépondérant pour le premier. Dans les deux cas, le commun peut être appréhendé comme un agir politique.

Les recherches sur les communs font apparaître la dimension politique de la propriété (Douai, 2014). Pour Béatrice Parance et Jacques De Saint Victor (2014), le passage d'un contexte historique "d'abondance et de progrès " à des "économies de pénurie » rendrait nécessaire l'élaboration d'autres modes plus inclusifs d'appropriation. En tant que construit, le commun relève d'un ensemble de choix politiques : «Ce qui relève du périmètre commun et/ou collectif et/ou public a son origine dans une décision de type politique, et ce quel que soit l'échelon où elle est prise, allant du local au global » (Harribey, 2011: 107). Olivier Weinstein insiste sur ce point. La formation d'un commun repose sur la " construction d'un système institutionnel » comme " ensemble cohérent de règles et de normes régissant les relations entre les individus impliqués dans l'usage et/ou la production d'un certain bien, et définissant les droits et obligations de ces membres, ce que l'on peut voir comme un véritable système politique spécifique » (Weinstein, 2015 : 73).

Pris comme principe d'action politique, le bien commun appelle à reconnaitre le pluralisme des valeurs (Walzer, 1983 ; Vatn, 2005). Cette idée est, par exemple, au cœur de la théorie de la justification de Luc Boltanski et Laurent Thévenot (1991:28), définie comme « une théorie de la justice qui tiendrait compte de la diversité des façons de spécifier le bien commun ». À chaque « cité » ou à chaque registre de justification correspond un bien commun en tant que principe selon lequel sont appréciés les actes, les choses et les personnes (Boltanski et Chiapello, 1999 : 163).

Les travaux de John R. Commons (2005 [1934]) permettent d'éclairer cette idée de bien commun comme projet politique à l'échelle d'un groupement humain plus ou moins vaste. Pour l'auteur, une action collective s'accompagne nécessairement d'une entente (concertée ou non) sur la nature du bien commun. Il pose le concept de «collectif dynamique organisé »[going concern $]^{14}$, expression qui lui permet de souligner la dimension active des groupements humains. Le groupement perdure tant que l'action collective est pérenne. Mais, il peut disparaître, se dissoudre ou être absorbé par un autre. Les individus peuvent le quitter, ou au contraire l'incorporer, si bien que le collectif peut perdurer tout en changeant de forme. Le collectif dynamique organisé est moins un groupement d'individus qu'un ensemble d'anticipations conjointes des membres qui le constituent (Buchs, 2016). Le bien commun représente ce qui est visé par l'action collective. Il en est le motif qui relie les personnes entre elles à un moment donné et les connecte avec un dessein, étant entendu que : "[les humains] vivent dans le futur mais agissent dans le présent » (Commons, 2005 [1934] : 84) ${ }^{15}$.

Trois articles du dossier thématique traitent, plus ou moins explicitement, de la dimension politique des communs. Celui de Rémi Barbier, Bernard Barraqué et Cécile Tindon (2019) s'inscrit dans les débats relatifs à la qualification de l'eau potable comme bien privé, public ou commun, voire comme droit humain, et les modes de gouvernance qui en découlent. Critiquant des approches économiques qui privilégient la dimension marchande de l'eau potable et se réfèrent à des catégorisations peu opérationnelles, les auteurs dépassent les analyses en termes de droits et proposent une perspective anthropologique originale. Celle-ci est fondée sur le concept d'« espace de coexistence » 
qui permettrait d'aller au-delà d'approches centrées sur l'accès et l'usage (matériel) pour prendre en compte les dimensions symboliques associées à un imaginaire social du commun (immatériel) qu'ils définissent. Le bien commun devient ici un référent pour guider l'action politique et le "vivre ensemble », au-delà d'une vision utilitariste de l'interdépendance entre individus (via un contrat social). Les auteurs concrétisent ensuite ces considérations théoriques en discutant les conditions d'intégration d'un réseau d'eau potable dans un "espace de coexistence». Ils en retiennent trois, lesquelles sont débattues dans l'article : l'indisponibilité au profit, l'accès pour tous et la démocratisation de la gouvernance, au cœur d'un projet politique qui permettrait de rattacher l'eau potable à la sphère du bien commun, c'est-à-dire à un monde à la fois matériel et immatériel. Ce dernier constituerait un espace de coexistence, spécifique en fonction des sociétés, des rapports de force et des époques.

De leur côté, Simone Ranocchiari et Christophe Mager (2019) s'appuient sur les expériences de projets de gestion commune de l'urbain à Bologne et à Naples (Italie). Ils développent une approche critique du concept de «bien commun» et arguent de la nécessité d'appréhender le commun en tant que pratique sociale et politique, le commoning, plutôt qu'en tant qu'objet. Les auteurs observent comment émerge et s'institutionnalise l'engagement de citoyens pour l'entretien de biens communs tels que des rues, des parcs, mais aussi des immeubles en état d'abandon ou de sous-utilisation. Les dimensions sociales et matérielles des communs permettent, dans les cas étudiés, d'éclairer des pratiques d'émancipation et d'autonomie des personnes et des collectifs. Ces pratiques se traduisent par la création de nouvelles communautés là où le manque d'espace public avait pu conduire au délitement des liens sociaux.

31 À travers l'analyse fine d'un projet de réhabilitation urbaine de deux places dites « traditionnelles » à Porto Novo (Bénin), Élizabeth Auclair et Élise Garcia (2019) discutent les dimensions matérielles et immatérielles, mais aussi visibles et invisibles, symboliques et émotionnelles, des places traditionnelles considérées comme « des communs » dans les villes béninoises. Elles détaillent la complexité de cette qualification dans un contexte où ces espaces combinent des dimensions privées, publiques et « communes " complexes à décrypter. Les outils de la géographie et de l'aménagement sont associés à une démarche anthropologique pour décoder les logiques sous-jacentes à ce projet, les leviers mobilisés et les blocages rencontrés. Ce projet pilote de réhabilitation est fondé, d'une part, sur la reconnaissance d'un système de valeurs plurielles pour orienter l'action publique et, d'autre part, sur la participation effective d'une pluralité d'acteurs tels que les habitants, les usagers, les artisans et les artistes. Leurs représentations et leurs usages de ces places sont intégrés à part entière dans la coconstruction de cette opération de réhabilitation, aux côtés des savoirs experts, notamment portés par les aménageurs. Les enjeux de patrimonialisation renvoient par ailleurs à l'identification et à la préservation de ces communs, à la reconnaissance des dimensions socio-économiques de ces espaces urbains, ce qui conduit les auteures à redéfinir la notion de patrimoine (au-delà des objets, la prise en compte des «communautés patrimoniales»), tout en insistant sur la dynamique du processus de patrimonialisation. Elles montrent que la mémoire, les traditions, les pratiques culturelles et rituelles [vodoun], donc la dimension immatérielle des communs, sont autant de formes collectives d'appartenance et d'attachement pouvant être considérées comme les signes de la construction d'un agir politique.

Avant de conclure, mentionnons que la contribution de Delphine Panossian (2019) à la rubrique «Points de vue» entre en résonance directe avec le dossier thématique. 
L'auteure dresse le bilan d'actions menées au sein du Parc naturel régional des Caps et Marais d'Opale qui procèdent d'un agir politique, en l'occurrence la construction collective d'un projet de territoire appréhendé comme un commun. Des modalités d'action collective participatives impliquant une grande variété d'acteurs (dont les habitants du parc), conjuguées à des initiatives originales faisant une large place à la création artistique, ont permis de mettre en lumière l'articulation des dimensions matérielles (milieu naturel et lieu d'activités économiques) et immatérielles du territoire du parc (patrimoine culturel, mémoire du territoire). L'auteure insiste sur la nécessité de mobiliser différents registres, des connaissances scientifiques et profanes aux ressentis émotionnels des habitants, comme autant de leviers d'action collective pour préserver et valoriser le territoire du parc constitué d'une variété de patrimoines (culturel, naturel et paysager).

$\mathrm{Au}$ final, les trois entrées retenues dans cette introduction permettent d'appréhender les liens entre les dimensions matérielles et immatérielles des communs, et ne sont pas exclusives les unes des autres. Au contraire, il est possible de les concevoir comme des facettes non exhaustives d'une même problématique liée à la complexité des communs. À ce titre, la problématique des communs (im)matériels appelle nécessairement un regard pluridisciplinaire, voire interdisciplinaire. Grâce à la diversité des objets traités et des corpus théoriques et conceptuels issus de disciplines variées, ce dossier thématique permet de recenser plusieurs pistes pour affiner la compréhension immatérielle des communs matériels et/ou de la dimension matérielle des communs immatériels, voire de montrer que cette dichotomie est artificielle.

\section{BIBLIOGRAPHIE}

Agrawal A., 2003, « Sustainable governance of common-pool resources : context, methods, and politics », Annual Review of Anthropology, vol. 32, p. 243-262.

Aigrain P., 2005, Cause commune : l'information entre bien commun et propriété, Paris, Fayard.

Allaire G., 2013, « Les communs comme infrastructure institutionnelle de l'économie marchande ", Revue de la régulation, vol. 14, $2^{\mathrm{e}}$ semestre.

Allaire G., 2019, «L'ambivalence des communs », Développement durable et territoires, vol. 10, $\mathrm{n}^{\circ} 1$, https://journals.openedition.org/developpementdurable/13442.

Aoki M., 2006, Fondements d'une analyse institutionnelle comparée, Paris, Albin Michel.

Aubriot O., Riaux J. (dir.), 2013, « Savoirs sur l'eau : techniques, pouvoirs », Autrepart, vol. 65.

Auclair É., Garcia É, 2019, « Les places traditionnelles de Porto-Novo (Bénin) comme communs : entre mise en valeur des espaces urbains et promotion des pratiques sociales et culturelles ", Développement durable et territoires, vol. 10, $\mathrm{n}^{\circ}$ 1, https://journals.openedition.org/ developpementdurable/13147. 
Bagdassarian I., Peneranda A., Baron C., 2019, « Évolution d'un commun d'altitude : entre matérialité et immatérialité ", Développement durable et territoires, vol. 10, n 1, https:// journals.openedition.org/developpementdurable/13377.

Baland J.-M., Platteau J.-P., 1996, Halting degradation of natural resources : is there a role for rural communities ?, Oxford, Clarendon Press.

Ballet J., 2007, «La gestion en commun des ressources naturelles : une perspective critique », Développement durable et territoires, varia (2004-2010), https://journals.openedition.org/ developpementdurable/3961.

Barbier R., Barraqué B., Tindon C., 2019, « L'eau potable pourrait-elle devenir un bien commun? ", Développement durable et territoires, vol. 10, n 1, https://journals.openedition.org/ developpementdurable/13231.

Baron C., Petit O., Romagny B., 2011, « Le courant des “common-pool resources" : un bilan critique », in Dahou T., Elloumi M., Molle F., Gassab M., Romagny B. (dir.), Pouvoirs, sociétés et nature au sud de la Méditerranée, Paris-Tunis, INRAT-IRD-Karthala, p. 29-52.

Boltanski L., Chiapello È., 1999, Le nouvel esprit du capitalisme, Paris, Gallimard.

Boltanski L., Thévenot L., 1991, De la justification. Les économies de la grandeur, Paris, Gallimard.

Bommier S., Renouard C., 2018, L'entreprise comme commun, Paris, Éditions Charles Léopold Mayer. Bromley D. W., 1989, « Property relations and economic development : the other land reform », World Development, vol. 17, $\mathrm{n}^{\circ}$ 6, p. 867-877.

Brondizio E. S., Pérez R., 2017, « L’École de Bloomington », in Cornu M., Orsi F., Rochfeld J. (dir.), Dictionnaire des biens communs, Paris, PUF, p. 474-477.

Buchanan J. M, 1965, « An economic theory of clubs », Economica, vol. 32, n 125, p. 1-14.

Buchs A., 2016, La pénurie en eau est-elle inéluctable?, Bruxelles, Berne, Peter Lang.

Buclet N., Cerceau J., 2019, « Interactions et rétroactions entre dimensions matérielle et immatérielle de systèmes communs de ressources spatialisés, une lecture par l'écologie territoriale ", Développement durable et territoires, vol. 10, n 1, https://journals.openedition.org/ developpementdurable/13467.

Calvo-Mendieta I., Petit O., Vivien F.-D., 2017, « Common patrimony : a concept to analyze collective natural resource management. The case of water management in France ", Ecological Economics, vol. 137, p. 126-132.

Chanteau J.-P., Labrousse A., 2013, «L'institutionnalisme méthodologique d'Elinor Ostrom : quelques enjeux et controverses ", Revue de la régulation, vol. 14, 2emestre.

Chavance B., 2012, «John Commons's organizational theory of institutions : a discussion ", Journal of Institutional Economics, vol. 8, n 1, p. 27-47.

Ciriacy-Wantrup S. von, Bishop R., 1975, « Common property as a concept in natural resources policy », Natural Resources Journal, vol. 15, p. 713-727.

Cole D. H., Ostrom E. (dir.), 2012, Property in land and other resources, Cambridge, Lincoln Institute of Land Policy.

Commons J. R., 2005 [1934], Institutional economics. Its place in political economy [3 $3^{\mathrm{e}}$ édition], New Brunswick, Transaction Publishers. 
Costanza R., Howarth R. B., Kubiszewski I., Liu S., Ma C., Plumecocq G., Stern D.I., 2016, « Influential publications in ecological economics revisited », Ecological Economics, vol. 123, p. 68-76.

Dacheux E., Goujon D., 2013, «Cohésion sociale et richesse économique : compléter l'apport d'Elinor Ostrom par une étude empirique de l'ESS », Management et Avenir, vol. 4, nº 65, p. 141-163.

Dardot P., Laval C., 2014, Commun : essai sur la révolution au XXI siècle, Paris, La Découverte.

Douai A, 2014, « De la dimension politique de la propriété et des institutions : apports et limites de l'approche d'E. Ostrom ", Revue internationale de droit économique, vol. 28, n 3, p. 301-317.

Frischmann B. M., 2012, Infrastructure. The social value of shared resources, Oxford, Oxford University Press.

Frischmann B. M., Madison M. J., Strandburg K. J (dir.), 2014, Governing the knowledge commons, Oxford, Oxford University Press.

Gand S., Segrestin B., 2009, « Peut-on partager la direction de l'entreprise ? Retour sur les entreprises démocratiques », Entreprises et Histoire, $n^{\circ}$ 57, p. 126-140.

Girard F., 2019, « Semences et agrobiodiversité : pour une lecture ontologique des bio-communs locaux », Développement durable et territoires, vol. 10, $\mathrm{n}^{\circ}$ 1, https://journals.openedition.org/ developpementdurable/13339.

Godelier M., 2010, L'idéel et le matériel. Pensée, économies, sociétés, Paris, Flammarion.

Harribey J.-M., 2011, « Le bien commun est une construction sociale. Apports et limites d'Elinor Ostrom », L’Économie politique, vol. 49, p. 98-112.

Hess C., Ostrom E., 2003, «Ideas, artifacts, and facilities : information as a common-pool resource ", Law and Contemporary Problems, vol. 66, p. 111-146.

Hess C., Ostrom E., 2007, Understanding knowldege as a commons, Cambridge, The MIT Press.

Larrère C., Larrère R., 2015, « Du bien commun au monde commun : la biodiversité ", in Larrère C., Larrère R., Penser et agir avec la nature. Une enquête philosophique. Paris, La Découverte, p. 263-278.

Leyronas S., Bambridge T., 2018, « Communs et développement : une approche renouvelée face aux défis mondiaux ", Revue internationale des études du développement, vol. 233, n 1, p. 11-29.

Linck T., 2012, «Économie et patrimonialisation. Les appropriations de l'immatériel », Développement durable et territoires, vol. 3, $\mathrm{n}^{\circ} 3$, https://journals.openedition.org/ developpementdurable/9506.

Locher F., 2013, « Les pâturages de la Guerre Froide : Garrett Hardin et la "Tragédie des communs" ", Revue d'histoire moderne \& contemporaine, vol. 60, p. 7-36.

McGinnis M. D., 2011, « An introduction to IAD and the language of the Ostrom workshop : a simple guide to a complex framework », The Policy Studies Journal, vol. 39, n 1, p. 169-183.

Musgrave R. A., 1957, « A multiple theory of budget determination », FinanzArchiv, vol. 17, n 3, p. 333-343.

Nahrath S., 2015, « Bien commun », in Bourg D., Papaux A. (dir.), Dictionnaire de la pensée écologique , Paris, PUF.

Netting R. McC, 1974, "The system nobody knows : village Irrigation in the Swiss Alps ", in Downing T.E., Gibson McG. (dir.), Irrigation's impact on society, Tucson, The University of Arizona Press, p. 67-76. 
Orsi F., 2013, « Elinor Ostrom et les faisceaux de droits : l'ouverture d'un nouvel espace pour penser la propriété commune ", Revue de la régulation, vol. $14,2^{\mathrm{e}}$ semestre.

Ostrom E., Schlager E., 1996, « The formation of property rights ", in Hanna S., Folke C., Mäler K.G. (dir.), Rights to nature, Washington DC, Island Press, p. 127-156.

Ostrom E., 1990, Governing the commons. The evolution of institutions for collective action, New York, Cambridge University Press.

Ostrom E., 2000, « Private and common property rights », in Bouckaert B., De Geest G. (dir.), Encyclopedia of Law and Economics (vol. II), Cheltenham, Edward Elgar, p. 332-379.

Ostrom E., 2005, Understanding institutional diversity, Princeton, Princeton University Press.

Ostrom E., 2010, « Beyond markets and States : polycentric governance of complex economic systems ", The American Economic Review, vol. 100, n 3, p. 641-672.

Ostrom V., Ostrom E., 1977, « Public goods and public choices », in Savas E.S. (dir.), Alternatives for delivering public services : toward improved performance, Boulder, Westview Press, p. 7-49.

Panossian D., 2019, « Transmettre le patrimoine comme un tout. Une initiative dans le Parc naturel régional des Caps et Marais d'Opale », Développement durable et territoires, vol. 10, $\mathrm{n}^{\circ} 1$, https://journals.openedition.org/developpementdurable/13817.

Parance B., De Saint Victor J., (dir.), 2014, Repenser les biens communs, Paris, CNRS Éditions.

Parsons K. H., 1950, « John R. Commons' point of view », in Commons J.R., The economics of collective action, New York, Macmillan Company, p. 341-375.

Petit O., Romagny B., 2009, « La reconnaissance de l'eau comme patrimoine commun : quels enjeux pour l'analyse économique? », Mondes en développement, vol. 145, p. 29-54.

Polanyi K., 1983 [1944], La grande transformation. Aux origines politiques et économiques de notre temps , Paris, Gallimard.

Ranocchiari S., Mager C., 2019, «Bologne et Naples au prisme des biens communs : pluralité et exemplarité de projets de gestion "commune" de l'urbain », Développement durable et territoires, vol. 10, $\mathrm{n}^{\circ}$ 1, https://journals.openedition.org/developpementdurable/13238.

Renou Y., Charmettant H., 2019, « Transformation coopérative, travail de négociation et communs : les interactions du matériel et de l'idéel ", Développement durable et territoires, vol. 10, $\mathrm{n}$ ${ }^{\circ} 1$, https://journals.openedition.org/developpementdurable/13299.

Samuelson P.A., 1954, « The pure theory of public expenditure », Review of Economics and Statistics, vol. $36, \mathrm{n}^{\circ} 4$, p. 387-389.

Sarker A., Blomquist W., 2018, Addressing misperceptions of Governing the Commons, Journal of Institutional Economics [pré-publication en ligne], p. 1-21.

Schlager E., Ostrom E., 1992, « Property-rights regimes and natural resources : a conceptual analysis ", Land Economics, vol. 68, n 3, p. 249-262.

Schweizer R., 2018, « Avoiding the commons trap : an exploration of local community governance of water in Valais, Switzerland », in Bréthaut C., Schweizer R. (dir.), A critical approach to international water management trends. Policy and practice, Londres, Palgrave Macmillan, p. 23-44.

Sen A., 2000, Un nouveau modèle économique. Développement, justice, liberté, Paris, Odile Jacob.

Tool M. R., 1977, « A social value theory in neoinstitutional economics », Journal of Economic Issues, vol. $11, \mathrm{n}^{\circ} 4$, p. 823-846. 
Vatn A., 2005, Institutions and the environment, Cheltenham, Edward Elgar.

Wade R., 1988, Village republics. Economic conditions for collective action in South India, Cambridge, Cambridge University Press.

Walzer M., 1983, Spheres of justice : a defence of pluralism and equality, Oxford, Robertson.

Weinstein O., 2013, « Comment comprendre les “communs" : Elinor Ostrom, la propriété et la nouvelle économie institutionnelle ", Revue de la régulation, vol. $14,2^{\mathrm{e}}$ semestre.

Weinstein O., 2015, « Comment se construisent les communs », in Coriat B. (dir.), Le retour des communs. La crise de l'idéologie propriétaire, Paris, Les liens qui libèrent, p. 69-86.

\section{NOTES}

1. Au $1^{\text {er }}$ décembre 2018, la bibliothèque virtuelle des communs [Digital library of the commons] de l'International Association for the Study of the Commons (IASC) compte plus de 10300 entrées, dont 3991 articles. À titre d'illustration, l'ouvrage d'Elinor Ostrom, Governing the commons : the evolution of institutions for collective action (1990), a été la deuxième publication la plus citée dans la revue Ecological Economics sur la période 2004-2014 (Costanza et al., 2016 : 70).

2. Journée d'étude "Action collective et communs", organisée le $1^{\mathrm{er}}$ avril 2016 à Sciences Po Toulouse par les coordinateurs de ce dossier. Malgré les nombreux ponts (conceptuels, théoriques et méthodologiques), la partition des communautés de chercheurs et des littératures afférentes, selon les communs considérés (matériels/environnementaux ou immatériels/ connaissances), était manifeste.

3. Vingt-sept propositions de contributions ont été recueillies (résumés détaillés). Compte tenu de leur qualité, la première sélection par le comité de coordination s'est avérée difficile. Et parmi les dix articles retenus et mis en évaluation, huit sont finalement publiés dans ce dossier.

4. Sous un angle historique (Locher, 2013; Brondizio et Pérez, 2017); sous un angle méthodologique (Chanteau et Labrousse, 2013) ; d'un point de vue critique (Agrawal, 2003 ; Baron et al., 2011 ; Harribey, 2011).

5. Devenue depuis 2006 l'International Association for the Study of the Commons (IASC).

6. Cette troisième confusion appelle à la nécessaire distinction «entre une réserve halieutique et les poissons pêchés, entre un jardin communautaire et les tomates qui y sont cueillies, entre un pommier et les pommes, entre une bibliothèque et les livres empruntés, etc. » (Allaire, 2013).

7. ["Increasingly being privatized, monitored, encrypted, and restricted"]. Ceci peut renvoyer à un phénomène d'enclosure (Aigrain, 2005) des communs de la connaissance faisant écho à celui qui avait affecté les pâturages anglais (Polanyi, 1944).

8. ["Common-pool resources may be owned by national, regional, or local governments; by communal groups; by private individuals or corporations; or used as open access resources by whomever can gain access."]

9. Cette question renvoie notamment aux travaux en termes de gouvernance polycentrique des systèmes complexes (Ostrom, 2010), à savoir des modes de gouvernance multiniveaux (du local au global), multitypes (juridictions imbriquées), multisectoriels (organisations et acteurs publics, privés, communautaires et hybrides), multifonctionnels (objectifs, modes de financement, modes de résolution des conflits, etc.) (McGinnis, 2011).

10. Une première version de ce tableau a été publiée en 1977 (Ostrom et Ostrom, 1977 : 12). Elle s'appuie sur les travaux de Paul Samuelson (1954) et de Richard Musgrave (1957) qui définissent les critères de non-rivalité et de non-exclusion et la notion de biens publics [merit goods], ainsi que sur ceux de James Buchanan (1965) pour la définition des biens de club. 
11. ["Exclusion relates to the difficulty of restricting those who benefit from the provision of a good or a service. Subtractability refers to the extent to which one individual's use subtracts from the availability of a good or service for consumption by others. Both of these two attributes can range from low to high."]

12. ['Transactions [...] are not the 'exchange of commodities', in the physical sense of 'delivery', they are the alienation and acquisition, between individuals, of the rights of future ownership of physical things, as determined by the collective working rules of society. The transfer of these rights must therefore be negotiated between the parties concerned, according to the working rules of society, before labour can produce, or consumers can consume, or commodities be physically delivered to other persons."]

13. La traduction des termes relatifs aux positions des acteurs n'étant pas stabilisée, nous présentons le tableau en anglais.

14. Un collectif dynamique organisé est une organisation d'activités coordonnées; c'est un comportement collectif avec un objectif commun et une volonté collective, régi par des règles opérantes communes (Parsons, 1950 : 355).

15. ["Indeed, it may be said that man lives in the future but acts in the present."]

\section{RÉSUMÉS}

Le dossier thématique "Communs (im)matériels » a pour objectif d'affiner la compréhension de la dimension immatérielle des communs matériels et/ou de la dimension matérielle des communs immatériels, voire de montrer que cette dichotomie est illusoire. Les objets traités dans ce dossier (l'espace urbain, l'eau, le territoire, les coopératives, les semences ou encore les estives) conjuguent des corpus théoriques et conceptuels variés reflétant la diversité des disciplines mobilisées (économie, géographie, aménagement, droit, gestion, anthropologie et sciences politiques). Nous avons choisi d'appréhender la conjugaison des dimensions matérielles et immatérielles des communs via trois entrées, non exclusives les unes des autres. La première, l'entrée épistémologique, insiste sur la complexité inhérente aux communs. Elle vise à questionner l'a priori selon lequel un objet particulier considéré comme un commun pourrait, par essence, être matériel ou immatériel, en clarifiant les définitions et les niveaux d'analyse des communs. La deuxième entrée, institutionnelle, conjugue les dimensions matérielles et immatérielles des communs par les enjeux liés à la définition et l'adoption de règles, en particulier celles relatives à la propriété, à la possession et à l'accès, mais également par les jeux d'acteurs qui y sont associés. La troisième entrée appréhende les liens entre les dimensions matérielles et immatérielles par les enjeux politiques inhérents aux communs, en insistant moins sur les communs comme systèmes de ressources que comme supports d'un agir politique. Ces trois entrées structurent l'articulation des articles qui composent ce dossier thématique.

The aim of the "(in)tangible commons" special section is to refine the understanding of the intangible dimension of tangible commons and/or the tangible dimension of intangible commons, or even to show that this dichotomy is illusory. The research objects (urban area, water, land, cooperatives, seeds, summer pastures) are analyzed thanks to various theoretical and conceptual corpora reflecting the diversity of the disciplines mobilized (economics, geography, planning, law, management, anthropology and political science). We have chosen to grasp the combination of the tangible and intangible dimensions of the commons through three perspectives, which are not exclusive of each other. The first, the epistemological perspective, emphasizes the inherent complexity of the commons and aims at questioning the a priori 
according to which a specific object considered as a commons could be tangible or intangible per se. The second perspective, institutional, combines the tangible and intangible dimensions of the commons through the issues of rules shaping, particularly those related to ownership, possession and access, but also through the linked coordination issues. The third perspective deals with the links between the tangible and intangible dimensions of the commons through the political issues at stake, regarding the commons as political projects. These three perspectives structure the articulation of articles within the special section.

\section{INDEX}

Keywords : tangible commons, intangible commons, property, resources, complexity, collective action, political project

Mots-clés : communs matériels, communs immatériels, propriété, ressources, complexité, action collective, projet politique

\section{AUTEURS}

\section{ARNAUD BUCHS}

Arnaud Buchs est maître de conférences en économie à l'université Toulouse Jean Jaurès et membre de l'équipe Dynamiques rurales du Laboratoire interdisciplinaire solidarités, sociétés, territoires (LISST-DR, UMR CNRS-EHESS-ENSFEA-UT2J). Ses recherches articulent une approche en économie écologique et institutionnaliste et le recours au terrain. Elles portent sur l'analyse des politiques et des règles qui encadrent la régulation des ressources en eau et leurs usages. arnaud.buchs@univ-tlse2.fr

\section{CATHERINE BARON}

Catherine Baron est professeure en aménagement de l'espace et urbanisme à Sciences Po Toulouse, et chercheure au Laboratoire d'étude et de recherche sur l'économie, les politiques et les systèmes sociaux (LEREPS, Sciences Po Toulouse, université de Toulouse). Ses travaux portent sur la gouvernance des eaux (ressources, services d'eau potable) avec une approche interdisciplinaire à la croisée de l'économie institutionnaliste, l'anthropologie du développement et l'aménagement. Ses terrains se situent en Afrique subsaharienne (essentiellement au Burkina Faso et au Niger) et en Indonésie.

baron@ut-capitole.fr

\section{GÉRALDINE FROGER}

Géraldine Froger est économiste, professeure à l'université Toulouse Jean Jaurès et directrice adjointe du Laboratoire d'étude et de recherche sur l'économie, les politiques et les systèmes sociaux (LEREPS, Sciences Po Toulouse, université de Toulouse). Ses recherches portent sur les politiques environnementales et de conservation de la biodiversité, en particulier dans les pays en développement, avec une approche combinant économie institutionnaliste et économie écologique.

geraldine.froger@univ-tlse2.fr

\section{ADRIEN PENERANDA}

Adrien Peneranda est maître de conférences en sciences de gestion à Sciences Po Toulouse et chercheur au Laboratoire d'étude et de recherche sur l'économie, les politiques et les systèmes 
sociaux (LEREPS, Sciences Po Toulouse, université de Toulouse). Ses travaux portent sur le management public et les collaborations public-commons autour de la diffusion numérique du patrimoine culturel.

adrien.peneranda@sciencespo-toulouse.fr 